\title{
Some Observations on Holographic Algorithms
}

\author{
Leslie G. Valiant* \\ School of Engineering and Applied Sciences \\ Harvard University \\ valiant@seas.harvard.edu
}

\begin{abstract}
We define the notion of diversity for families of finite functions, and express the limitations of a simple class of holographic algorithms in terms of limitations on diversity. We go on to describe polynomial time holographic algorithms for computing the parity of the following quantities for degree three planar undirected graphs: the number of 3-colorings up to permutation of colors, the number of connected vertex covers, and the number of induced forests or feedback vertex sets. In each case the parity can be computed for any slice of the problem, in particular for colorings where the first color is used a certain number of times, or where the connected vertex cover, feedback set or induced forest has a certain number of nodes. These holographic algorithms use bases of three components, rather than two.
\end{abstract}

\section{Introduction}

The theory of holographic algorithms is based on a notion of reduction that enables computational problems to be interrelated with unusual fluidity. The theory offers three basic reduction techniques:

(a) Holographic transformations that relate pairs of problems by simply taking a different view or basis,

(b) Holographic gadgets that use internal cancelations custom designed for the problems at hand, and

(c) Interpolation techniques for recovering information from the outputs of computations on a set of specially prepared variants of the problem instance at hand.

The overarching open question in the theory is whether this combination of techniques can bridge the gap between classical polynomial algorithms on the one hand, and the class of \#P-complete (or NP- or $\oplus \mathrm{P}$-complete) problems as defined by classical reductions, on the other.

In order to further our understanding of this question we introduce here the notion of diversity for finite functions, in terms of which some limitations of the simplest kinds of holographic algorithms that we discussed in an earlier paper [V06] can be explored more explicitly. These simplest holographic algorithms are those obtained from what we define as elementary reductions. We show that

\footnotetext{
* This work was supported in part by NSF-CCF-04-27129.
} 
such algorithms do impose a limitation on the diversity of the functions that can be realized. It remains unresolved, however, whether holographic algorithms that are not bound by the constraints of elementarity, such as those given in later sections of this paper, can evade this diversity limitation.

In the later sections we go on to describe some polynomial time holographic algorithms for three natural problems for undirected graphs of degree three. These compute the parity of the number of solutions of each of the following three problems: feedback vertex sets (or, equivalently, induced forests), connected vertex covers, and vertex 3-colorings up to permutations of colors.

Besides evading the elementarity constraint our algorithms have other features that put them outside the currently better understood regions of holographic theory. For one thing the use of the three element basis b3 from [V08] puts them outside the collapse theorem of Cai and $\mathrm{Lu}$ [CL09], and hence outside any known classification such as [CL07]. (Of course, the possibility has not yet been excluded that \#P-complete problems can be solved even within the scope of this collapse theorem or classification.) Second, the results hold for parity rather than counting. For parity problems, or fixed finite fields in general, holographic transformations and interpolation both appear to offer less flexibility than they do for general counting problems. For example, understanding the complexity of the counting problems modulo three, for the structures we analyze here modulo two, appears to remain a challenge.

For brevity of exposition we shall assume familiarity with the basic notions and notations of holographic algorithms as described in ([V04], [V08]).

\section{Diversity}

For a Boolean function $f\left(x_{1}, \ldots, x_{m}\right)$ and a subset $S \subseteq X=\left\{x_{1}, \ldots, x_{m}\right\}$ of size $n$, we define the diversity of $S$ in $f$ to be the logarithm to the base two of the number of different functions of the $n$ variables of $S$ that can be obtained by fixing the $m-n$ remaining variables $X-S$ in the $2^{m-n}$ different ways. This is the central concept in Neciporuk's proofs [N66] of lower bounds on formula complexity. He showed that if $X$ has a partition into subsets $S_{i}$ such that the average diversity of the $S_{i}$ in $f$ is substantial, then a nonlinear lower bound on the formula size of $f$ follows.

We say that a Boolean function $f\left(x_{1}, \ldots, x_{m}\right)$ has $n$-diversity $D$ if $D$ is the maximum diversity of $S$ in $f$, over all subsets $S \subseteq X=\left\{x_{1}, \ldots, x_{m}\right\}$ of size $n$. Since there are $2^{2^{n}}$ Boolean functions of $n$ variables, the maximum $n$-diversity of a function is $2^{n}$.

A Boolean function family $f=\left\{f_{m}\left(x_{1}, \ldots, x_{m}\right) \mid m=1, \ldots\right\}$ has diversity $g(n)$ if for each positive integer $n, g(n)$ is the maximum $n$-diversity of $f_{m}$ for any $m \geq n$. Clearly $g(n) \leq 2^{n}$.

A Boolean function family $f$ has polynomial diversity if its diversity $g(n)$ is upper bounded by some polynomial $p(n)$. It has exponential diversity if it is lower bounded by $c 2^{c n^{\kappa}}$ for some constants $c, \kappa>0$. It has exponential standard diversity if, for some polynomial $p(n)$, exponential diversity is achieved for all $n$ 
by $f_{m}$ with some $m \leq 2^{p(n)}$. It has polynomial standard diversity if, for all polynomials $p(n)$, the $n$-diversity achieved for $n$ by $f_{m}$ with $m \leq 2^{p(n)}$ is polynomial bounded.

Such definitions can also be made for finite fields $F_{q}$ for families with $f_{i}$ : $\{0, \ldots, q-1\}^{i} \mapsto\{0, \ldots, q-1\}$. In that case the maximum $n$-diversity of a family is $q^{n} \log _{2} q$.

High diversity does not imply high complexity. The Circuit Value problem [L75] $\mathrm{CV}_{n, r}\left(x_{1}, \ldots, x_{n+r}\right)$ we shall formulate here as the function that regards its first $n$ inputs as a vector $v$ of $n$ Boolean values, and the remaining bits as a specification of a Boolean circuit $C$ of $n$ inputs with binary gates. An $m$ gate circuit of $n$ inputs can be specified using $r=\mathrm{O}((n+m) \log (n+m))$ binary bits. Since all the $2^{2^{n}}$ Boolean functions of $n$ variables can be realized by a circuit with $\mathrm{O}\left(2^{n} / n\right)$ gates [Lu58], they can all be encoded in $\mathrm{CV}_{n, r}$ if $r=\mathrm{O}\left(2^{n}\right)$. We define $\mathrm{CV}$ to have such an encoding of circuits. Hence CV has diversity $2^{n}$ since $S=\left(x_{1}, \ldots, x_{n}\right)$ has diversity $2^{n}$ in $\mathrm{CV}_{n, r}$ for an appropriate $r=\mathrm{O}\left(2^{n}\right)$. Clearly $\mathrm{CV}$ then also has exponential standard diversity.

Using the following notion of reduction one can deduce that most natural $\mathrm{P}-$, NP- and \#P-complete problems have exponential standard diversity. We say that a reduction $\tau$ from $\left\{\mathrm{CV}_{n, r}\right\}$ to a family of functions $\left\{Q_{i}\right\}$ is segregating if in polynomial time $\tau$ maps the pair $v, C$ to a pair of Boolean sequences $(y, z)$ such that (i) for any fixed $n$ and $r$, the lengths of $y$ and of $z$ are uniquely determined, (ii) the length of $y$ is polynomially bounded in terms of $n$, (iii) $y$ depends on $v$ and not $C$, (iv) $z$ depends on $C$ and not $v$, and (v) $Q_{i}(y, z)=C(v)$. (In short, $y$ encodes $v, z$ encodes $C$, and $Q_{i}$ evaluates $C$ on $v$. The length of $y$ is polynomial in $n$, but the length of $z$ may be exponential in $n$.)

Proposition 1 If $C V$ is reducible to $Q$ by a segregating reduction then $Q$ has exponential standard diversity.

Proof For a fixed size $n$ consider $\mathrm{CV}_{n, r}$ with $r$ exponential in $n$ and large enough that all $2^{2^{n}}$ Boolean functions of $n$ variables can be expressed. Now consider one of the $2^{2^{n}}$ choices of $C$. Since the reduction, say $\tau$, is segregating, for all $v$ it will map $(v, C)$ to $(y, z)$ for the some fixed value of $z$. For $C$ and $z$ so fixed, as $v$ varies so will $y$, and $Q_{i}(y, z)=C(v)$. Hence, $Q_{i}$ will compute on the encoding $y$ of $v$ the same Boolean function as $C$ does on $v$. Hence, fixing $z$ in different ways will make $Q_{i}$ compute $2^{2^{n}}$ different functions of $y$. If $S$ is the set of variables that represents $y$ then the diversity of $S$ in $Q_{i}$ will be $2^{n}$. Since $\tau$ is segregating, by condition (ii) $|S|$ is polynomially bounded in terms of $n$. It follows that the diversity of $S$ in $Q_{i}$ will be at least $c 2^{c|S|^{1 / \kappa}}$ for appropriate positive constants $c$ and $\kappa$.

Now for many NP-complete problems, by tracing through the known reductions, one can derive segregating reductions from $\mathrm{CV}$ to them. For example, consider the family $Q$ corresponding to Cook's 3SAT problem. Here $Q_{i}(x)$ is a $3 \mathrm{CNF}$ formula with $i$ clauses and variables from $x_{1}, \ldots, x_{i}$. From a circuit $C$ with inputs $x_{1}, \ldots, x_{n}$, and any vector $v$ of values of $x_{1}, \ldots, x_{n}$, one can construct by 
now standard methods a polynomial size 3CNF formula that is satisfiable if and only if that circuit $C$ on that input $v$ evaluates to one: The formula will have the first $n$ clauses encode the input with the $j^{\text {th }}$ clause being $\left(x_{j}\right)$ or $\left(x_{j}^{\prime}\right)$ according to whether the $j^{\text {th }}$ among the $n$ bits of $v$ is 1 or 0 . It will have the remaining clauses encode the gates. This is a segregating polynomial time reduction from $\mathrm{CV}$ to $3 \mathrm{CNF}$. Related to the $3 \mathrm{CNF}$ satisfiability problem is $\oplus 3 \mathrm{CNF}$, the problem of determining the parity of the number of solutions of a $3 \mathrm{CNF}$ formula, and their planar analogs $\mathrm{Pl}-3 \mathrm{CNF}$ and $\oplus \mathrm{Pl}-3 \mathrm{CNF}$. From the above construction we can deduce the following.

Proposition 2 The problems $3 C N F, \oplus 3 C N F, P l-3 C N F$ and $\oplus P l-3 C N F$ all have exponential standard diversity.

Proof The previous paragraph describes a segregating reduction from CV to $3 \mathrm{CNF}$. This establishes the result for $3 \mathrm{CNF}$ by virtue of Proposition 1 . Since the construction can be made to preserve the number of solutions, the 3CNF formula will have 0 or 1 solutions according to whether the value output by the circuit $C$ is 0 or 1 . The result for $\oplus 3 \mathrm{CNF}$ therefore also follows. For the planar case one uses additional sets of clauses that act as crossovers and make the formula planar, as described by Lichtenstein [L82]. These can also be made to preserve the number of solutions [HMRS98]. These additional clauses can be viewed as part of the circuit encoding, and then yield a segregating reduction to the planar versions of $\mathrm{Pl}-3 \mathrm{CNF}$ and $\oplus \mathrm{Pl}-3 \mathrm{CNF}$ as needed.

With this starting point one can ask for each of the known NP-complete problems, such as those of Karp [K72], whether CV is reducible to some natural encodings of them by a segregating reduction. It appears that this is the case for the vast majority, and for those it then follows that some natural encoding of them has exponential standard diversity.

What is the status of the numerous counting problems that are known to be complete in an appropriate counting class, but for which existence is polynomial time computable and not known to be complete for P? Do these counting problems have CV embedded in them equally explicitly? The following shows that in some such cases the embedding is in fact explicit.

Proposition 3 The Permanent modulo $k$ for any prime $k \neq 2$ has exponential standard diversity.

Proof From the proof in [V79a] one can obtain a segregating reduction from $\mathrm{CV}$ via $3 \mathrm{CNF}$ to the permanent modulo $k$ for any prime $k$ other than two.

On the other hand, if the proof of \#P-completeness goes through interpolation (eg [V79b], [J87], [Vad01], [XZZ07], [CLX08]) then exponential diversity does not appear to follow immediately. For example, does counting matchings modulo 3 in some natural encoding of planar graphs [J87], have exponential diversity? There are cases in which the known reductions to the counting problem take particularly circuitous routes through interpolations, raising the possibility 
that the $\mathrm{CV}$ problem is truly disguised, but nevertheless exponential diversity can be deduced from known reductions for the corresponding parity problem. One example of this is planar vertex cover for which known \#P completeness proofs [Vad01, XZZ07] are indirect. However, for the subclass of planar 3/2bipartite graphs (bipartite graphs with degree 2 on one side and 3 on the other) a segregating reduction from $3 \mathrm{CNF}$ to this vertex cover problem that preserves the parity of the number of solutions can be derived from the the $\oplus \mathrm{P}$-completeness proof of this problem given in [V06].

Proposition 4 The parity of the number of vertex covers for planar $3 / 2$ graphs has exponential standard diversity.

Clearly a unary Boolean function family, one that is zero whenever any $x_{i}=1$, will have polynomial diversity. Since there exist unary functions of arbitrarily high Turing machine time complexity it follows that polynomial diversity does not imply polynomial time Turing machine complexity. Pavel Pudlak has made the following elegant observation that shows that low diversity can also be possessed by functions that have high complexity in many other senses, such as having exponential circuit complexity or being NP-complete. For any function $f(x)$ on $n$ inputs consider an error correcting code $g:\{0,1\}^{n} \rightarrow\{0,1\}^{r}$ that corrects more than $n$ errors and can be computed and inverted efficiently. Let $h:\{0,1\}^{r} \rightarrow\{0,1\}$ be such that $h(y)=1$ iff $y=g(x)$ for some $x$ and $f(x)=1$. Then $h$ has $n$-diversity at most $n+1$ since for any domain $d$ of $n$ of its input bits, fixing the remaining bits will permit it to have value 1 for at most 1 of the $2^{n}$ values of $d$, and hence there are at most $2^{n}+1$ such different functions possible of the $n$ variables of $d$.

\section{Elementary Reductions to Matchgrids}

We shall now define the notion of an elementary reduction to matchgrids. The definition is a generalization of the one given in [V06] that was specific to reductions from 3CNF. Here we consider a family $f$ with respect to input domain $d$. This means that for each $m$ there is a specified function $f_{m}$ on Boolean variables $x_{1}, \ldots, x_{m}$, and a specified subset $d_{m}$ of $n_{m}$ of these $m$ variables.

We say that $\tau$ is a $k(m)$-oracle reduction if for family member $f_{m}$ it generates $k(m)$ matchgrids in polynomial time and from their Holants it computes the solution to the original problem also in polynomial time. Note that while many holographic algorithms in the literature are 1-oracle, some multi-oracle matchgrid reductions that use interpolation have been described also ([V04], [V08], [CC07]).

Suppose that $\tau$ is a polynomial time 1-oracle reduction from $f$ to matchgrids over field $F$. For a function $f_{m}$, a domain of its variables $d_{m}$ of size $n=n_{m}$, and an assignment $z$ to the set $c_{m}$ of variables that is the complement of $d_{m}$, let $\mathrm{M}\left(f_{m}, d_{m}, z\right)$ be the set of $2^{n}$ adjacency matrices of the set of matchgrid images 
under $\tau$ of the $2^{n}$ restrictions of $f_{m}$ when the $n=n_{m}$ variables specified by $d_{m}$ are fixed in all possible ways.

Then $\tau$ is a local boundary reduction for $\left(f_{m}, d_{m}\right)$ if for each $m, z$ and adjacency matrix in $\mathrm{M}\left(f_{m}, d_{m}, z\right)$ there is a planar embedding such that for every fixed $m$ and $z$

(a) the corresponding $2^{n}$ embeddings have an identical set of nodes $U_{m, z}$.

(b) in these $2^{n}$ embeddings all the edges and their weights are identical, except possibly those that have both endpoints within a subset $Z_{m, z} \subseteq U_{m, z}$, which is of size upper bounded by a polynomial $L(n)$ independent of $m$.

(c) the $Z_{m, z}$ nodes have degrees bounded by a constant independent of $n, m$ or $z$.

(d) the nodes $Z_{m, z}$ all lie in the infinite outer face of the embedding of the graph induced by $T_{m, z}=U_{m, z}-Z_{m, z}$, and

(e) the edges incident to pairs of vertices in $Z_{m, z}$ can be partitioned into $n$ sets such that each such set $S_{i}$ corresponds to a variable $x_{k_{i}}$ in $d_{m}$, and the weights of $S_{i}$ are functions of the value of $x_{k_{i}}$ but are independent of the values of the other $x_{j}$ in $d_{m}$ (i. e. those with $j \neq k_{i}$.)

We shall say that a reduction $\tau$ from a family $f$ with respect to input domain $d$ of size $n$ to matchgrids over $F$ is elementary if it has the four properties of (i) being 1-oracle, (ii) being local boundary, (iii) having the number of members $|F|$ polynomial bounded in $m$, and (iv) having Holant $(\tau(f(x)))$ determine the value of $f(x)$. We then also say that $\tau$ is an elementary reduction for $(f, d)$.

We note that there are no constraints on what the transformation does on different $z$. The intent is that when the Circuit Value problem is embedded, then the different circuits $C$ can be embedded in arbitrarily different ways, but for any one circuit there are constraints on the way the matchgrids can vary as the inputs $v$ to $C$ vary. Also note that the field size is allowed to grow polynomially with $m$, so that it can be exponential in $n$ if $m$ is exponential in $n$.

\section{Elementary Reductions Compute Functions of Polynomial Diversity}

Our negative results are based on the following statement [V06]:

Theorem 1 There is a weighted planar graph $G$ having $r$ external nodes and $O\left(r^{4}\right)$ edges of which all but $O\left(r^{2}\right)$ have fixed weight 1 , such that for any field $F$, any $r$-component standard signature that is realized by some matchgrid can be realized by $G$ by setting the $O\left(r^{2}\right)$ variable-weight edges to appropriate constants. Proof For $r \leq 4$ this result is proved in [V08] (Propositions 6.1-6.3). For general $r$ the corresponding result was proved by Cai, Choudhary and Lu (Corollary 4.1 in [CCL09]), but for matchcircuits. The general result then follows from the equivalence of matchgrids and matchcircuits (Lemmas 3.1 and 3.2 in [CC07]).

Theorem 2 For any family $f$ and domain $d$ if there is an elementary reduction to matchgrids for $(f, d)$, then $d$ has polynomial standard diversity in family $f$. 
Proof Suppose that $\tau$ is a polynomial time 1-oracle reduction from $f$ to matchgrids over field $F$. For a function $f_{m}$ and a domain of its variables $d_{m}$ of size $n=n_{m}$, for each $z$ let $\mathrm{M}\left(f_{m}, d_{m}, z\right)$ be the set of adjacency matrices as defined above and consider the planar embeddings that respect conditions (a)-(e) of the definition of elementarity.

By (a), (b) these embeddings are identical with respect to all the edges that are incident to a $T_{m, z}$ node at laest at one end. We regard the embedding of the nodes $T_{m, z}$ as a matchgrid $H_{m, z}$. By (d) the remaining nodes $Z_{m, z}$ are all mapped into the outer face of $H_{m, z}$. Since, by (b) and (c), $\left|Z_{m, z}\right|$ is upper bounded by a polynomial $L(n)$, and the degrees of the $Z_{m, z}$ nodes by a constant, $H_{m, z}$ has $\mathrm{O}(L(n))$ external connections, and can be regarded as a matchgrid with $\mathrm{O}(L(n))$ external nodes. Now, by Theorem $1, H_{m, z}$ can be replaced by a matchgrid with $\mathrm{O}\left((L(n))^{2}\right)$ variable weight edges. From this we deduce that as $z$ varies, the total number of inequivalent matchgrids $H_{m, z}$ is at most $a_{1}=|F|^{O\left((L(n))^{2}\right)}$. (In other words it is single exponential in $n$ however large $m$ may be.)

It remains to complete the estimation of the number of different functions of the original $n$ domain $d_{m}$ variables that the matchgrids can realize as $z$ varies, by also taking into account the remainder of the matchgrid specification, namely the nodes $\left|Z_{m, z}\right|$ and the edges incident to them. We can fix the names of the nodes of $Z_{m, z}$ and the external nodes of $H_{m, z}$, which altogether number $\mathrm{O}(L(n))$. Then the number of potential edges that have at least one endpoint in $\left|Z_{m, z}\right|$ is at most $A=\mathrm{O}\left((L(n))^{2}\right)$. By assumption (e), each choice of $z$ partitions the edges among pairs of $Z_{m, z}$ nodes into $n$ sets, and each such set will have a weight assignment that represents the corresponding domain variable having value zero, and a weight assignment corresponding to value one. The number of partitions is upper bounded by $a_{2}=A^{n}$ clearly. Also, for each such partition, $a_{3}=|F|^{2 A}$ upper bounds the number of distinct values of the edges between $Z_{m, z}$ nodes that among them represent all combinations of representing 0's and 1's for the $n$ variables of $d_{m}$. Also, the number of possible weight assignments to edges incident to both $\left|Z_{m, z}\right|$ and $\left|T_{m, z}\right|$ nodes is upper bounded by $a_{4}=|F|^{O(L(n))}$. It follows that the total number of functions of the domain variables that the matchgrids can realize is upper bounded by $a_{1} a_{2} a_{3} a_{4}$, which itself is upper bounded by $(L(n)|F|)^{O\left((L(n))^{2}\right)}$. Now, by condition (iii) of elementarity, $|F|$ is polynomial bounded in $m$. For standard diversity $m$ is single exponential in a polynomial $p(n)$. It follows that the number of distinct functions is at most $2^{O(q(n))}$ for some polynomial $q$. In other words the standard diversity is at most polynomial in $n$.

From this result one can deduce for problems known to have high diversity that they do not have elementary reductions to matchgrids. The following is an instance that parallels a result in [V06]:

Corollary 1 There is no elementary reduction from $(f, d)$ to matchgrids where $f$ is any one of $\mathrm{Pl}-3 \mathrm{CNF}, 3 \mathrm{CNF}, \oplus \mathrm{Pl}-3 \mathrm{CNF}$ or $\oplus 3 \mathrm{CNF}$, and $d$ specifies a subset of $\mathrm{O}(\log m)$ of the clauses for formulae with $m$ clauses.

Proof This follows from Proposition 2 and Theorem 2. 
It is an interesting question whether converse implications also hold. For problems such as $\#_{7} \mathrm{Pl}$-Rtw-Mon-3CNF [V06] for which 1-oracle holographic algorithms exist, even for fields whose size does not increase at all with the input size, one would like to determine whether they have polynomial diversity. For planar representations of Boolean functions one can define a notion of planar diversity where the domains $d_{m}$ have to be on the outer face of the embedding. Then the particulars of the algorithm just described for $\#_{7} \mathrm{Pl}-\mathrm{Rtw}-\mathrm{Mon}-3 \mathrm{CNF}$ do imply polynomial planar diversity for that problem. However, such arguments do not appear to apply to domains that are not on the periphery, or to general diversity.

Multiple oracle calls appear to be very useful in reductions among counting problems. There are multitudes of \#P-complete problems that have been proved complete via reductions that involve multiple oracle calls and polynomial interpolation on the results ([V79b], [J87], [Vad01], [XZZ07], [CLX08]). For any one of these problems one can ask whether they have polynomial diversity.

In the opposite direction, one can ask whether algorithms that make multiple oracle calls, each via an elementary reduction, can compute functions of exponential diversity. To formulate specific questions of this kind one would need to define specific classes of such multiple oracle call algorithms. One relevant such class is offered by the algorithms described in Sections 6-8 of this paper. These all have the following form: Given an instance $G$ of the problem, one generates a single matchgrid with weights that are polynomials in $x$ with coefficients from a field $F$. The solution sought is the $j$ th least significant bit in the coefficient of $x^{i}$ of the Holant, where $i, j$ are predetermined integers, and all the coefficients are guaranteed to be integral. There is the further constraint that if this coefficient is nonzero then it has at least $j-1$ factors of 2 . Note that the solutions here are obtained by the multi-oracle reduction that evaluates the matchgrid at enough different values of $x$, and then interpolates for the appropriate coefficient. It is an interesting question to determine whether or not these classes of reductions can evade the polynomial constraint on diversity of elementary reductions.

\section{The basis b3}

The basis $\mathbf{b 3}$ [V08] has three components $\mathbf{z}=(1,0), \mathbf{n}=(1,-1), \mathbf{p}=(1,1)$. It has the useful property that for all $x \in F, \mathrm{xz}^{3}+\mathbf{n}^{3}+\mathbf{p}^{3}$ is an even ternary signature and therefore, by Proposition 6.2 in [V08], is realizable by a planar matchgate. To verify this it is sufficient to expand $x \mathbf{z}^{3}+\mathbf{n}^{3}+\mathbf{p}^{3}$ as :

$(x, 0,0,0,0,0,0,0)+(1,-1,-1,1,-1,1,1,-1)+(1,1,1,1,1,1,1,1)$

$=(x+2,0,0,2,0,2,2,0)=[x+2,0,2,0]$.

We shall call this signature, and the gate realizing it, $\mathbf{g}_{3}(x)$. The analogous two-output signature $\mathbf{g}_{2}(x)$ is also even, and therefore realizable by virtue of Proposition 6.1 in [V08], since

$x \mathbf{z}^{2}+\mathbf{n}^{2}+\mathbf{p}^{2}=(x, 0,0,0)+(1,-1,-1,1)+(1,1,1,1)=[x+2,0,2]$, as is also the one output signature $\mathbf{g}_{1}(x)=x \mathbf{z}+\mathbf{n}+\mathbf{p}=[x+2,0]$ 
For each of the three parity problems that we define in the sections that follow, we shall consider planar graphs of $n$ vertices all of maximum degree three. Our constructions do not require that the graph be cubic in the sense that every node has degree exactly three.

For each problem we shall construct for any such graph $G$ a family of matchgrids $\Omega(G, x)$ indexed by $x$, using a fixed binary recognizer $\mathbf{r}$ for the edges, and the above mentioned generators $\mathbf{g}_{1}(x), \mathbf{g}_{2}(x)$ and $\mathbf{g}_{3}(x)$, for the nodes of degrees one, two and three, respectively. Then for each problem, Holant $(\Omega(G, x))$ can be viewed as a polynomial in $x$ of degree at most $n$. If we evaluate $\operatorname{Holant}(\Omega(G, x))$ for one $G$ and $n+1$ distinct values of $x$, and interpolate for the coefficients, then the coefficient of $x^{i}$ will be the sum of the contributions to the Holant of the states in which exactly $i$ of the generators are generating z's, and the remainder n's or p's.

Alternatively, we shall sometimes substitute $\mathbf{g}_{1}(s), \mathbf{g}_{2}(t)$ and $\mathbf{g}_{3}(x)$, with different indeterminates $s, t, x$. Then after evaluating at $\mathrm{O}\left(n^{3}\right)$ distinct points, we can interpolate to obtain the coefficient of $s^{i} t^{j} x^{l}$, which gives the contribution to the Holant of states where among the $\mathbf{z}$ generators, exactly $i$ have degree one, $j$ degree two, and $l$ degree three.

We now describe the binary recognizers that we use for the edges. Each of these recognizers is a simple chain, of one, two or three edges, with the end nodes serving as the two external nodes. In our notation below * denotes a node, and $*(w)^{*}$ denotes an edge of weight $w$ between two nodes. The following can be verified by inspection.

Proposition 5 The values of the following three recognizers are as follows when $(a, b)$ is input from the left, and $(c, d)$ from the right:

$\mathbf{r 1}: *(1) *$ has value $a c+b d$,

$\mathbf{r 2 :} *(1)^{*}(1)^{*}$ has value $a d+b c$, and

r3: * $(1) *(-1)^{*}$ has value ad - bc.

\section{Holographic Algorithm for the Parity of the Number of Induced Forests or Feedback Vertex Sets}

The Minimum Feedback Vertex Set problem for undirected graphs is defined as follows: Given an undirected graph $G$ and an integer $k$ the question is to determine whether there is a set of $k$ vertices whose removal leaves a forest (i.e. a graph with no cycles.) There is a substantial literature on this existence problem. The directed version of this problem was proved NP-complete by Karp [K72]. The undirected version we study here was proved NP-complete by Garey and Johnson [GJ79]. Subsequently it was shown to be NP-complete even for planar graphs of degree four by Speckenmeyer [S83]. For cubic (i.e regular degree three) graphs a polynomial algorithm was given by Li and Liu [LL99]. (This last result is also implied by the polynomial time algorithm of Ueno et al. [UKG88] for the Minimum Connected Vertex Cover problem (defined in the next section) for cubic graphs, in conjunction with the result of Speckenmeyer that for any cubic 
graph on $n$ vertices MCVC-MFVS $=n / 2-1$, where MCVC and MFVS denote the sizes of the minimum connected vertex cover and the minimum feedback vertex set.)

Here we are interested not in the existence problem but in the parity of the number of solutions, not only for forests of the largest size but for forests of every size, and not only for regular graphs of degree three, but for all graphs of maximum degree three. Thus the probem we address, $\oplus m F V S$, is the following: Given a degree $m$ undirected graph $G$ and an integer $k$, determine the parity of the number of sets of $k$ nodes that induce a forest in $G$.

Theorem 3 There is a deterministic polynomial time algorithm for $\oplus 3 F V S$.

Proof We place $\mathbf{g}_{3}(\mathbf{x}), \mathbf{g}_{2}(\mathbf{x})$ and $\mathbf{g}_{1}(\mathbf{x})$ generators at vertices of degree three, two and one respectively. We place a recognizer $\mathbf{r} \mathbf{1}$ on each edge. Then, by Proposition 5, the value of each recognizer as a function of the nine possible combinations of what the adjacent nodes generate are as follows: $\mathbf{z z} \rightarrow 1$; $\mathbf{z p} \rightarrow$ $1 ; \mathbf{z n} \rightarrow 1 ; \mathbf{p z} \rightarrow 1 ; \mathbf{n z} \rightarrow 1 ; \mathbf{p p} \rightarrow 2 ; \mathbf{n n} \rightarrow 2 ; \mathbf{p n} \rightarrow 0 ; \mathbf{n p} \rightarrow 0$.

We regard each state $\sigma$ (i.e. each combination of states of all the generators) of the matchgrid as a two-coloring, where one color, Z, corresponds to the nodes generating z's, and the other, Y, those generating n's and p's. For each such state we define \# $Y Y(\sigma)$ to be the number of edges joining a pair of nodes both colored $\mathrm{Y}$, and \# Ycomponents $(\sigma)$ to be the number of connected components induced in $G$ by the removal of the $\mathrm{Z}$ nodes and the edges adjacent to them. Then the Holant will be the sum over all such Z/Y 2-colorings of $G$ of the value $V=2^{\# Y \text { components }(\sigma)+\# Y Y(\sigma)}$, since each connected component has one of two states (all $\mathbf{n}$ or all $\mathbf{p}$ ), and each edge in such a component contributes a further factor of two. If $G$ has $n$ nodes and the number of $\mathrm{Z}$ nodes is fixed as $n-k$, then the minimum number of divisors of 2 in $V$ is $2^{n-(n-k)}=2^{k}$, and is achieved if and only if the YY edges induce a forest in $G$. (Note that in any graph with $k$ nodes the sum of the number of edges and the number of connected components is at least $k$, the minimum being achieved only if the graph is a forest.) Hence, if one divides the coefficient of $x^{n-k}$ in Holant $(\Omega(G, x))$ by $2^{k}$, then the parity of that number is the desired solution to $\oplus 3 \mathrm{FVS}(G)$.

\section{Holographic Algorithm for the Parity of the Number of Connected Vertex Covers}

The Minimum Connected Vertex Cover problem is the following. Given an undirected graph $G$ determine the size of the smallest set of nodes that (i) is a vertex cover, and (ii) induces a connected subgraph of $G$.

The existence problem was shown NP-complete for degree four planar graphs by Garey and Johnson [GJ77]. Fernau and Manlove [FN06] showed that this result holds even in the bipartite case. For cubic graphs it was shown to be polynomial time computable by Ueno, Kajitani and Gotoh [UKG88].

Here we are interested in the following parity problem $\oplus m C V C$. Given an undirected planar graph $G$ of maximum degree $m$ and an integer $k$, the problem 
is to compute the parity of the number of connected vertex covers of $G$ of $k$ vertices.

Theorem 4 There is a deterministic polynomial time algorithm for $\oplus 3 C V C$. Proof We place $\mathbf{g}_{3}(\mathbf{x}), \mathbf{g}_{2}(\mathbf{t})$, and $\mathbf{g}_{1}(\mathbf{s})$ generators at vertices of degree three, two and one respectively. We place a recognizer r2 on each edge. Then, by Proposition 5, the value of each recognizer as a function of the nine possible combinations of what the adjacent nodes generate are as follows: $\mathbf{z z} \rightarrow 0 ; \mathbf{z p} \rightarrow$ $1 ; \mathbf{z n} \rightarrow-1 ; \mathbf{p z} \rightarrow 1 ; \mathbf{n z} \rightarrow-1 ; \mathbf{p p} \rightarrow 2 ; \mathbf{n n} \rightarrow-2 ; \mathbf{p n} \rightarrow 0 ; \mathbf{n p} \rightarrow 0$.

As before, we regard each state $\sigma$ of the matchgrid as a two coloring, where one color, Z, corresponds to the nodes generating z's, and the other, Y, those generating n's and p's. For each such state we define \#YY $(\sigma)$ to be the number of edges joining a pair of nodes both colored $\mathrm{Y}$, and \#Ycomponents $(\sigma)$ to be the number of connected components induced in $G$ by these YY edges. Now the Holant will be the sum over some such Z/Y 2-colorings of $G$ in which the nodes colored $\mathrm{Z}$ form an independent set, of $V= \pm 2 \# Y \operatorname{components}(\sigma)+\# Y Y(\sigma)$. This will follow by a similar argument to that used in Theorem 3, except now the $\mathrm{Z}$ nodes form an independent set since the value of $\mathbf{r} \mathbf{2}$ for $\mathbf{z z}$ input is zero, and we need to analyze potential cancelations.

To derive this value of $V$ we first note that if the graph has $n$ nodes and is cubic, then for a state in which the $\mathrm{Z}$ nodes form an independent set of size $n-k$, it will be the case that $\# Y Y(\sigma)=3 n / 2-3(n-k)=3(k-n / 2)$. For each $\mathrm{Y} / \mathrm{Z}$ coloring and for any connected component induced by the $\mathrm{Y}$ colored edges in $G$, there will be two valid states, corresponding to the Y-colored nodes having all $\mathbf{p}$ or all $\mathbf{n}$ states. When one changes all the $\mathrm{Y}$ nodes from $\mathbf{p}$ to $\mathbf{n}$ then the values of all the recognizers in $G$ will change sign. Hence if the nodes in this component have an even number of edges incident to them then these contributions to the Holant will have the same sign, and otherwise will cancel. Hence the minimum (nonzero) number of divisors of 2 in $V$ is $2^{3(k-n / 2)+1}$, and is achieved if and only if the YY edges induce one connected component in $G$ and $G$ has an even number of edges. Hence, if one divides the coefficient of $x^{n-k}$ in Holant $(\Omega(G, x))$ by $2^{3(k-n / 2)+1}$, then the parity of that number is the desired solution to $\oplus 3 \mathrm{CVC}(G)$.

If the graph is not regular, then by interpolation we can find the coefficient of $s^{i} t^{j} x^{l}$ in Holant $(\Omega(G, s, t, x))$ for all $i, j, l$. For any specific combination of $i, j, l$ the value of $\# \mathrm{YY}(\sigma)$ is $|E|-i-2 j-3 l$, where $|E|$ is the total number of edges in $G$. Hence we can compute the parity of the number of solutions for any combination $i, j, l$, and hence for all the combinations with $i+j+l=n-k$. We shall derive the parity of the number of solutions corresponding to such $Z$ sets by dividing the appropriate coefficient by $2^{|E|-i-2 j-3 l+1}$ rather than by $2^{3(k-n / 2)+1}$ as used in the regular case.

So far we have assumed that the number of edges in $G$ is even. To treat the alternative case we choose an arbitrary edge and replace $\mathbf{r} \mathbf{2}$ by $\mathbf{r} \mathbf{1}$ on it. This ensures that when switching between all $\mathbf{p}$ and all $\mathbf{n}$ states the sign will not change on this one edge, and hence not for the product of all of these odd 
number of edges. It only remains to ensure that the $\mathrm{Y}$ nodes still form a vertex cover, and for this it is necessary to preclude that the endpoints of the chosen edge be both in state $\mathrm{Z}$. This can be done by multiplying the $x$ term in these two generators by a new indeterminate $w$, and, by interpolation, computing and adding the coefficients of $w^{0}$ and $w^{1}$ (while ignoring that of $w^{2}$.)

\section{Holographic Algorithm for the Parity of the Number of Vertex Colorings}

A 3-Vertex Coloring of a graph $G$ is an assignment of a color from a palette of 3 colors to each vertex so that no pair of adjacent vertices has the same color. Clearly the set of all such proper colorings can be partitioned into equivalence classes of 3 ! colorings, so that the members of each class differ only by a permutation of the colors. Here we are interested in the following two closely related problems. The problem $\oplus m$ Col: for an undirected planar graph $G$ of maximum degree $m$ determine the parity of the number of equivalence classes of 3-colorings of $G$. The problem $\oplus m F C o l$ : for an undirected planar graph $G$ of maximum degree $m$ and an integer $k$ determine the parity of the number of 3-colorings that are invariant under permutations of the remaining two colors when exactly $k$ nodes are given the first color. We note that the corresponding counting problems for 3-colorability of degree three graphs are \#P-complete [BDGJ99].

Theorem 5 [B04] For some constant $m, \oplus m$ Col is $\oplus P$-complete.

Theorem 6 There is a deterministic polynomial time algorithm for $\oplus 3 F C$ Col and for $\oplus 3$ Col.

Proof We place $\mathbf{g}_{3}(\mathbf{x}), \mathbf{g}_{2}(\mathbf{t})$, and $\mathbf{g}_{1}(\mathbf{s})$ generators at vertices of degree three, two and one respectively, and $\mathbf{r} 3$ recognizers on each edge. The $\mathbf{r} \mathbf{3}$ recognizers for $a d-b c$ are not symmetric, and can be placed in arbitrary orientation without influencing our result. By Proposition 5 the value of each recognizer as a function of the nine possible combinations of what the adjacent nodes generate are as follows: $\mathbf{z z} \rightarrow 0 ; \mathbf{z p} \rightarrow 1 ; \mathbf{z n} \rightarrow-1 ; \mathbf{p z} \rightarrow-1 ; \mathbf{n z} \rightarrow 1 ; \mathbf{p p} \rightarrow 0 ; \mathbf{n n} \rightarrow 0 ; \mathbf{p n} \rightarrow$ $-2 ; \mathbf{n p} \rightarrow 2$.

Again we regard each state $\sigma$ of the matchgrid as a two coloring, where one color, Z, corresponds to the nodes generating z's, and the other, Y, those generating n's and p's. For each such state we define \#YY $(\sigma)$ to be the number of edges joining a pair of nodes both colored $\mathrm{Y}$, and \#Ycomponents $(\sigma)$ to be the number of connected components induced in $G$ by these YY edges. Then the Holant will be the sum over some such Z/Y 2-colorings of $G$ in which the nodes colored $\mathrm{Z}$ form an independent set and those colored $\mathrm{Y}$ form a bipartite graph, of the values $V= \pm 2^{\# Y \text { components }(\sigma)+\# Y Y(\sigma)}$.

To see this we first assume that the graph is cubic and has an even number of edges. If the graph has $n$ nodes, then for a state in which the $\mathrm{Z}$ nodes form an independent set of size $k$, then $\# Y Y(\sigma)=3 n / 2-3 k$. We note that for each $\mathrm{Y} / \mathrm{Z}$ coloring the $Y Y$ edges will form a set of connected bipartite components in 
$G$. In each component there will be two valid states, corresponding to which of the two parts is in $\mathbf{p}$ or $\mathbf{n}$ state. When one swaps $\mathbf{p}$ and $\mathbf{n}$ all the values of all the recognizers will change sign. Hence if there are an even number of edges incident to the nodes in one such component, then the contributions to the Holant will have the same sign for the two states. Hence the minimum number of divisors of 2 in $V$ is $2^{3 n / 2-3 k+1}$, and is achieved if and only if the $\mathrm{YY}$ edges induce one connected bipartite component in $G$. Hence, if one divides the coefficient of $x^{k}$ in Holant $(\Omega(G, x))$ by $2^{3 n / 2-3 k+1}$, then the parity of that number is the parity of the number of solutions to $\oplus 3 \mathrm{FCol}(G)$.

Graphs that are not regular or have an odd number of edges can be treated exactly as in Theorem 4 .

For completeness we mention the following application:

Theorem 7 The parity of the number of edge 3-colorings of planar 3/2 bipartite graphs can be computed in polynomial time.

Proof The line graph of such a graph is a planar degree three graph, and hence the result follows immediately from Theorem 6 . Note that we do not require the $3 / 2$-graph to be a regular $3 / 2$ graph.

\section{References}

[B03] R. Barbanchon, Reductions fines entre problèmes NP-complets, PhD Thesis, Université de Caen Basse-Normandie, (2003).

[B04] R. Barbanchon, On unique graph 3-colorability and parsimonious reductions in the plane. Theoretical Computer Science, Volume 319, Issues 1-3, (2004) 455-482.

[BDGJ99] R. Bubley, M. Dyer, C. Greenhill and M. Jerrum, On approximately counting colourings of small degree graphs, SIAM J. Comput. 29 (1999), pp. 387-400.

[CC07] J-Y. Cai, and V. Choudhary, Some Results on Matchgates and Holographic Algorithms. International Journal of Software and Informatics, 2007,1(1):3 36.

[CCL09] J-Y. Cai, V. Choudhary and P. Lu, On the Theory of Matchgate Computations. Theory of Computing Systems, 45:1 (2009) p108-132.

[CL07] J-Y. Cai, P. Lu: Holographic algorithms: from art to science. STOC 2007 401410.

[CL09] J-Y. Cai, and P. Lu, Holographic algorithms: The power of dimensionality resolved. Theor. Comput. Sci. 410:18 (2009) 1618-1628.

[CLX08] J-Y. Cai, P. Lu, and M. Xia. Holographic Algorithms by Fibonacci Gates and Holographic Reductions for Hardness. FOCS (2008) 644-653.

[C71] S.A. Cook, The complexity of theorem proving procedures, Proceedings of the 3rd ACM STOC, (1971) 151-158.

[EGM09] B. Escoffier, L. Gourves, J. Monnot, Complexity and approximation results for the connected vertex cover problem in graphs and hypergraphs. Journal of Discrete Algorithms, 2009.

[FM06] H. Fernau and D. Manlove. Vertex and edge covers with clustering properties: Complexity and algorithms. Journal of Discrete Algorithms, 2009.

[GJ77] M. R. Garey and D. S. Johnson. The rectilinear steiner tree problem is NP complete. SIAM Journal of Applied Mathematics, 32:826834, 1977. 
[GJ79] M. R. Garey and D. S. Johnson. Computer and Intractability: A Guide to the Theory of NP-Completeness. W. H. Freeman, (1979).

[HMRS98] H. B. Hunt III, M. V. Marathe, V. Radhakrishnan, R. E. Stearns, The Complexity of Planar Counting Problems. SIAM J. Comput. 27(4) (1998) 1142-1167.

[J87] M. R. Jerrum, Two-dimensional monomer-dimer systems are computationally intractable. J. Statist. Phys. 48:1-2 (1987) 121-134.

[K72] R. M. Karp, Reducibility among combinatorial problems, in Complexity of Computer Computations, R.E. Miller and J.W. Thatcher, eds., Plenum Press (1972) 85104.

[L75] R. E. Ladner, The Circuit Value Problem is Log Space Complete for P. SIGACT NEWS, 7:1 (1975) 18-20.

[LL99] D. M. Li and Y. P. Liu, A polynomial algorithm for finding the minimum feedback vertex set of a 3-regular simple graph. Acta Math. Sci. 19:4 (1999) 375-381.

[Li82] D. Lichtenstein, Planar formulae and their uses. SIAM J. Comput. 11 (1982) 329-343

[Lu58] O. B. Lupanov, A method of circuit synthesis. Izv. VUZ Radiofiz 1, (1958) 120-140.

[N66] E. I. Neciporuk, A Boolean Function. Sov. Math. Dokl. 7, (1966) 999-1000.

[S83] E. Speckenmeyer, Untersuchungen zum Feedback Vertex Set Problem in ungerichteten Graphen. PhD Thesis, Universität Paderborn (1983).

[S88] E. Speckenmeyer, On feedback vertex sets and nonseparating independent sets in cubic graphs, Journal of Graph Theory, Volume 12, Issue 3 (1988) 405-412.

[UKG88] S. Ueno, Y. Kajitani, and S. Gotoh. On the nonseparating independent set problem and feedback set problem for graphs with no vertex degree exceeding three. Discrete Mathematics, 72:355-360, 1988.

[V79a] L. G. Valiant. The complexity of computing the permanent. Theoretical Computer Science, 8 (1979) 189- 201.

[V79b] L. G. Valiant. The complexity of enumeration and reliability problems. SIAM J. Computing, 8:3 (1979), pp.410-421.

[V04] L. G. Valiant. Holographic algorithms (extended abstract), Proc. 45th Annual IEEE Symposium on Foundations of Computer Science, Oct 17-19, Rome, Italy, (2004). IEEE Press, 306-315.

[V05] L. G. Valiant. Completeness for parity problems, Proc. 11th International Computing and Combinatorics Conference, Aug 16-19, Kunming, China, LNCS, Vol. 3959, (2005), Springer-Verlag, 1-9.

[V06] L. G. Valiant. Accidental algorithms, Proc. 47th Annual IEEE Symposium on Foundations of Computer Science, Oct 22-24, Berkeley, CA, IEEE Press, (2006), 509-517.

[V08] L. G. Valiant. Holographic algorithms, SIAM J. on Computing, 37:5, (2008) 1565-1594. (Earlier version: Electronic Colloquium on Computational Complexity, Report TR05-099, (2005).)

[XZZ07] M. Xia, P. Zhang, W. Zhao: Computational complexity of counting problems on 3-regular planar graphs. Theor. Comput. Sci. 384(1) (2007) 111-125. 